\title{
EDAD DE LAS LAVAS DEL MIEMBRO LOS BAMBINOS Y SUMARIO CRONOESTRATIGRÁFICO DE LA FORMACIÓN BARVA, COSTA RICA
}

\author{
Sandra G. Arredondo Li \& Gerardo J. Soto* \\ Tecnoambiente Centroamericano S.A., Apdo. 1488-1000 San José, Costa Rica \\ * Autor para contacto, dirección actual: Área de Amenazas y Auscultación Sísmica \\ y Volcánica (A $\left.{ }^{3} \mathrm{SV}\right)$, Instituto Costarricense de Electricidad, Apdo. 10032-1000, San \\ José, Costa Rica, correo-e: katomirodriguez@yahoo.com
}

(Recibido 20/02/07; aceptado 11/06/07)

\begin{abstract}
The age of Los Bambinos Member is established based on new geological observations, geology of drilled wells in the upper part of Barva volcano, and previous and a new radiometric ages. We also propose a new chronostratigraphy for Barva Formation (equivalent to Neo Barva volcano). The rocks underlying Tiribí Formation near the top of the volcano are stratigraphically correlationable to Colima Formation and are part of the Paleo Barva. Lavas from Bermúdez Member form several lava fields with a wide range of ages (270-40? ka). The pyroclastic deposits in stratovolcanoes like Barva are lesser in volume and are grouped diachronically, as it is the case of Carbonal Member, which in the upper part of the volcano can represent ages in the range 40-27.4 ka. Lavas of Los Bambinos Member are divided in two sub-members (Lower and Upper), and a lahar interbeded between the two sub-members yield an age of $27.4 \mathrm{ka}$. The whole member would have ages between 10-30 ka. The pyroclasts of Porrosatí Member could have ages $<15 \mathrm{ka}$, according to ages of tephras overlying Los Bambinos lavas.
\end{abstract}

Key words: Los Bambinos Member, Barva volcano, Barva Formation, chronostratigraphy, lavas.

RESUMEN: Basados en nuevas observaciones geológicas, geología de pozos en la parte alta del volcán Barva, y dataciones previas y una nueva, se establece la edad del Miembro Los Bambinos y se propone un cuadro cronoestratigráfico de la Formación Barva (equivalente al volcán Neo Barva). Las rocas subyacentes a la Formación Tiribí cerca de la cima son correlacionables estratigráficamente con la Formación Colima y forman parte del Paleo Barva, incluyendo a la Formación Tiribí. Las lavas del Miembro Bermúdez representan varios campos de coladas de lavas con un rango amplio de edades (270-40? ka). Los depósitos piroclásticos en los estratovolcanes como el Barva están subordinados en volumen y se agrupan diacrónicamente, como el caso del Miembro Carbonal, que en la parte superior del volcán puede tener edades en un rango de 40-27,4 ka. Las lavas del Miembro Los Bambinos se han dividido en dos sub-miembros (Inferior y Superior). Un lahar intercalado entre ambos sub-miembros muestra una edad de 27,4 ka. El miembro podría tener en total un rango de 10-30 ka. Los piroclastos del Miembro Porrosatí tendrían una edad $<15 \mathrm{ka}$, con base en la edad de tefras que sobreyacen a las lavas de Los Bambinos.

Palabras clave: Miembro Los Bambinos, volcán Barva, Formación Barva, cronoestratigrafía, lavas.

ARREDONDO, S.G. \& SOTO, G.J., 2006: Edad de las lavas del Miembro Los Bambinos y sumario cronoestratigráfico de la Formación Barva, Costa Rica.- Rev. Geol. Amér. Central, 34-35: 59-71. 


\section{INTRODUCCIÓN}

Las rocas de lo que hoy se denomina Formación Barva han sido descritas desde hace tres cuartos de siglo (Schaufelberger, 1932), aunque su estratigrafía no ha sido estudiada sino hasta hace apenas un par de décadas (Protti, 1986). Muchas de sus particularidades geovulcanológicas, estratigráficas y particularmente cronoestratigráficas, aún permanecen oscuras o poco claras. En parte se debe a la falta de un mapeo geológico detallado en el flanco sur del volcán Barva, y a que gran parte de las rocas del macizo volcánico se encuentra en medio de una selva de difícil acceso que cubre todo el sector septentrional del volcán y aun parte de su sector meridional, donde solo se han intentado mapeos principalmente fotogeológicos (vgr. Soto, 1999).

El objetivo de este trabajo es conjuntar datos dispersos de la geología, mapeo geológico, geología de pozos, estratigrafía y edades absolutas recientemente obtenidas de diferentes fuentes, para dilucidar primero la edad y secuencia estratigráfica de las lavas Los Bambinos, luego construir un sumario cronoestratigráfico de la Formación Barva tradicionalmente conocida en el sector sur del volcán, y al final hacer una propuesta de mayor alcance al grueso del macizo, incluyendo su flanco norte.

La metodología aplicada ha sido encaminada a reinterpretar la geología de algunos pozos y piezómetros claves y perfiles geofísicos en el sector alto meridional del volcán, conjuntado con un mapeo de campo y fotogeológico-geomorfológico de parte de ese sector, la recopilación, reinterpretación y calibración de edades absolutas (publicadas y una nueva de radiocarbono), y finalmente, una interpolación y extrapolación de unidades y edades, para proponer una evolución global del macizo del Barva.

\section{SÍNTESIS ESTRATIGRÁFICA DE LA FORMACIÓN BARVA}

En la primera mitad del siglo XX, Schaufelberger (1932) y Crosby (1945) describieron coladas de lava cerca de San Antonio de Belén, que luego Dengo \& Chaverri (1951) agruparon con el nombre de "Lavas de Ciruelas y Cebadilla". Williams (1952) las llamó "Lavas de Post Avalancha" por sobreyacer a los "Depósitos de Avalancha Ardiente", que son las ignimbritas de la Formación Tiribí. Bohnenberger (1968) describió esas mismas lavas como "Flujos de Lava Recientes", incluyendo al "Flujo Cebadilla" y el de "El Aeropuerto El Coco", complementado con un perfil longitudinal (Bohnenberger \& Madrigal, 1968), con la posición estratigráfica de esos flujos lávicos. Fernández (1969) fue quien finalmente definió la Formación Barva, agrupando no solo a las coladas del volcán Barva, sino también a las de San Rafael, San Antonio, Ciruelas y Cebadilla. Echandi (1981) escribió que las lavas provienen del macizo del Barva y de otros focos al sureste, donde alcanzan espesores máximos de $80 \mathrm{~m}$. Protti (1986) publicó un trabajo de la geología del flanco sur del volcán Barva y utilizó el nombre de Formación Barva para agrupar todos los tipos litológicos que constituyen el estratovolcán (ver Fig. 1A). La subdividió informalmente en seis unidades litológicas (en concordancia con el mapa hidrogeológico de BGS \& SENARA, 1985): miembros Bermúdez, Carbonal, Los Bambinos, Porrosatí, Los Ángeles y Cráter (Fig. 1). La edad estimada que asigna Protti (1986) al estratovolcán Barva es de 30 ka, y su origen lo interpreta a partir de una o varias fisuras regionales de la Cordillera Central.

Denyer \& Arias (1991) describieron la formación dentro de su mapa geológico de la Hoja Abra (Denyer \& Arias, 1990), como formada por lavas y piroclastos y le asignaron una edad de final del Pleistoceno al Holoceno. Pérez (2000) relaciona el origen de las ignimbritas de la Formación Tiribí (con una edad de $330 \mathrm{ka}$ ) con una caldera en la cima del Barva, e indica que las rocas más antiguas de la Formación Barva, que afloran en las cercanías de San Antonio de Belén, tienen una edad de 270 ka. Más recientemente, Gans et al. (2003) han datado las rocas de la Formación Colima Superior, que subyace a la Formación Tiribí, en 330 ka, y Pérez et al. (2006) han datado con mejor precisión las ignimbritas de la Formación Tiribí en 322 ka. Brenes (2003) cartografió las unidades del Barva, con nuevos nombres, en parte con la nomenclatura que había utilizado Protti (1986), pero funcionalmente, 
las propuestas de este último y BGS-SENARA (1985) tienen un mejor fundamento estratigráfico. Brenes (2003) aporta una datación C-14 de tefras que sobreyacen a las lavas Los Bambinos, con una edad de $11380 \pm 278$ años. Esta edad la hemos calibrado con base en Stuiver \& Reimer (2006) y se obtiene una edad calibrada de 13,4 $\pm 0,4 \mathrm{ka}$.

\section{ESTRATIGRAFÍA Y EDAD DE LAS LAVAS LOS BAMBINOS EN LA CUENCA ALTA DEL RÍO CIRUELAS}

Con base en una reevaluación de fotografías aéreas, mapas topográficos, morfología y los trabajos de Protti (1986) y Soto (1994), se propone que la extensión del Miembro Los Bambinos o Barva Superior es ostensiblemente diferente a la propuesta del primer autor, como se muestra en la figura 1. Además, se ha utilizado la geología del pozo-piezómetro BA-809 (231110 N - 524310 E), en la margen izquierda del río Ciruelas (Arredondo, 2003), como parámetro de comparación con la geología de los alrededores (Fig. 1 y 2) y la de pozos adyacentes perforados años atrás.

Las lavas del Miembro Los Bambinos afloran desde el norte de San Miguel en el camino hacia Porrosatí y de allí hacia el norte, este y oeste, y son blocosas con geoformas de aspecto joven. Según Soto (1994), con base en un mapeo fotogeológico y geomorfológico, estas lavas fueron eruptadas desde los conos del lado oriental de la cima del Barva, y también fluyeron hacia el sector norte y noreste, sobre el área de la caldera superior del volcán, con una distribución mostrada en la figura 1B. Las rocas son andesitas porfiríticas, con $30-40 \%$ de fenocristales, la mayoría (25-35\%) de plagioclasas característicamente entre $6-8 \mathrm{~mm}$, además de piroxenos $(<5 \%)$ y olivino aislado $(\sim 1 \%)$, en una matriz intersertal con microlitos y vidrio gris oscuro, en la que la cantidad de vesículas y el grado de oxidación son variables.

Esta unidad es un campo varias coladas superpuestas, con brechas autoclásticas en el piso y el techo, que pueden alcanzar $>100 \mathrm{~m}$ de espesor en el sector Montaña La Isla, cerca de la cima (233N-525E, aprox.). Desde el punto de vista morfológico e hidrogeológico, en el sector de Porrosatí, las coladas de lava forman dos unidades, que hemos denominado Los Bambinos Inferior y Los Bambinos Superior (Figs. 2 y 3). Desde el cruce de camino que va a Sacramento y Porrosatí y de allí hacia el norte, se observa una morfología con un fuerte escarpe morfológico de hasta 50 metros de alto, con pendientes que cambian de $8-10^{\circ}$ a $20-40^{\circ}$, que marca el frente de las coladas de Los Bambinos Superior, que se extiende hacia el oeste, pasa por la toma de la Fuente 29 (231050N-524250E, administrada por la Empresa de Servicios Públicos de Heredia, ESPH), y de allí unos $3 \mathrm{~km}$ al noroeste. La composición petrográfica de las lavas de ambas unidades es similar, reconocibles por las plagioclasas grandes $(<8 \mathrm{~mm})$ y pocos piroxenos. La relación espacial de ambos sub-miembros se explica como dos sub-campos de coladas de lavas, superpuesto parcialmente uno al otro (Fig. 3). Además, con base en la correlación geológica de los datos geofísicos mostrados por GeoStratu Consultores S.A. (2005), se observa que en medio de ambas unidades, hay un paquete de lahares que se acuña hacia el este, y que se han incluido en el Miembro Carbonal. Esto demuestra que el concepto estratigráfico de este miembro es de índole de facies volcánicas proximales-medias, y diacrónico, de modo que incluye tobas entre los Miembros Bermúdez y Bambinos, así como epiclastos entre las unidades del Miembro Bambinos.

Protti (1986) definió al Miembro Carbonal como una unidad "constituida por tobas líticas y tobas de ceniza poco consolidadas", entre los miembros Los Bambinos y Bermúdez, separando a Barva Superior e Inferior. Por lo visto en el río Ciruelas, en las perforaciones, y en la reinterpretación geológica de la geofísica, deben incluirse además lahares y otros epiclastos subyacentes al Miembro Los Bambinos y entre las unidades de este miembro. Estos epiclastos aparecen en medio de las unidades Los Bambinos Inferior y Superior, de modo que en su conjunto el Miembro Carbonal es un miembro diacrónico, que incluye varias etapas de formación. En el río Ciruelas afloran en la base de la catarata adyacente a la toma de la Fuente 29 (231050N524250 E; Figs. 3 y 4). En este punto se observan al menos $3 \mathrm{~m}$ de un lahar tipo debris flow, constituido por bloques decimétricos a métricos (cerca de 40\%) en una matriz café limo-arcillosa 


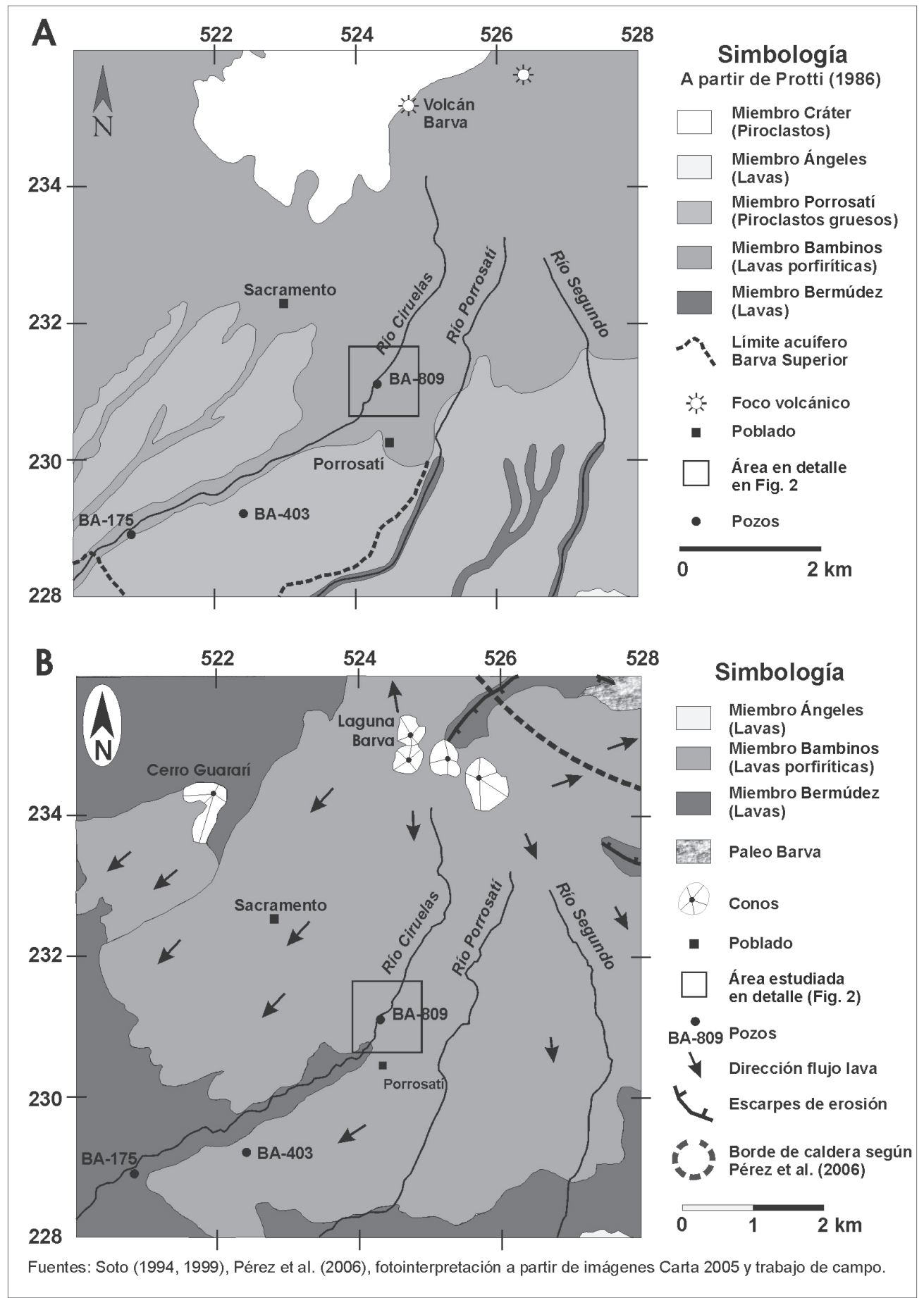

Fig. 1: Geología de la parte superior del flanco sur del volcán Barva. A: Redibujado de Protti (1986) con su detalle estratigráfico. B: La propuesta de este trabajo que obvia la cobertura de cenizas y muestra la distribución de los cuerpos lávicos y las estructuras volcánicas, estructurales y morfológicas principales. El cuadrado indica el área con geología en detalle de la figura 2. Se muestran los pozos utilizados para la correlación estratigráfica. 


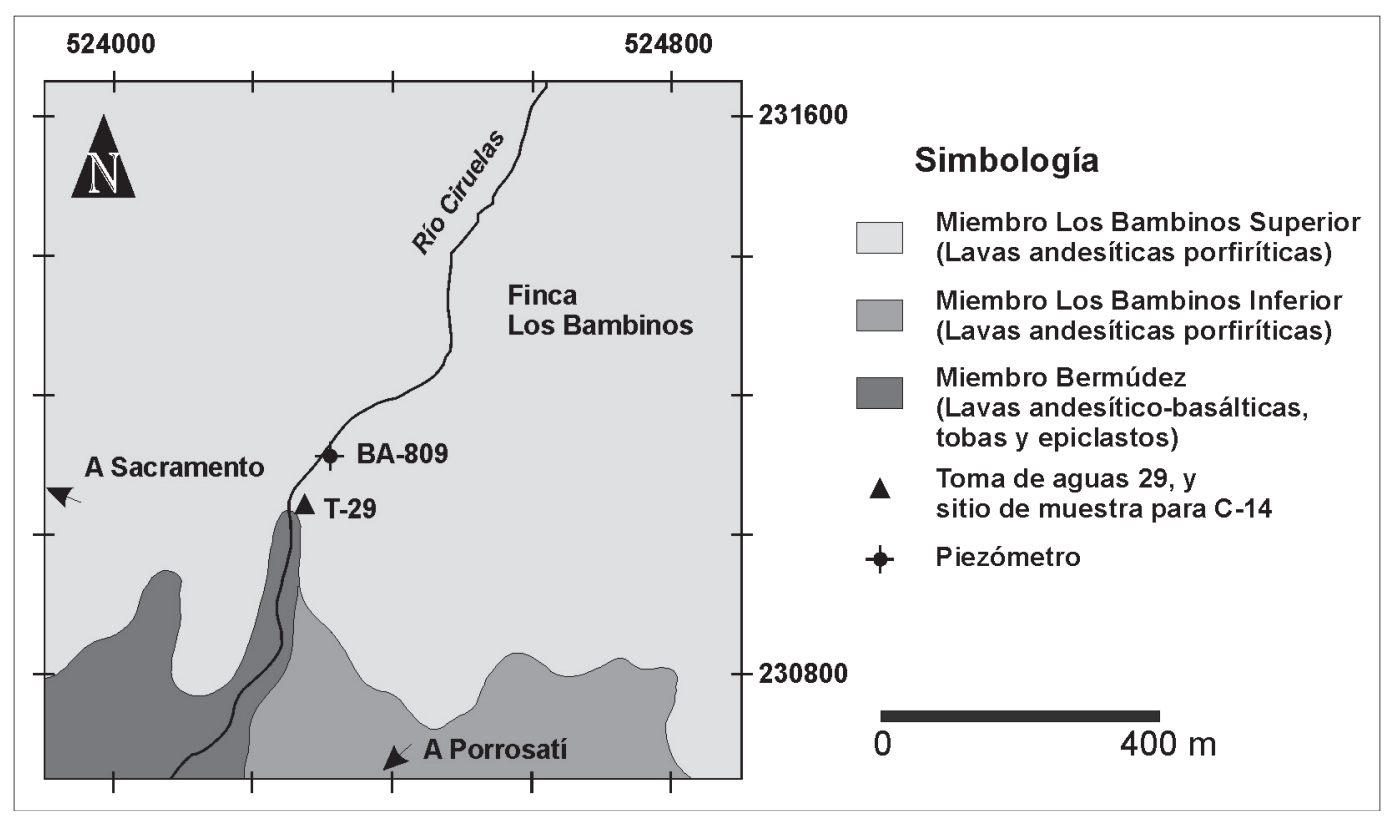

Fig. 2: Mapa geológico de parte de la cuenca alta del río Ciruelas.

de cenizas y fragmentos de lava. Sin embargo, en el pozo BA-403 alcanzan hasta $15 \mathrm{~m}$ de espesor total (Fig. 5). Estos cambios de espesor de los epiclastos son típicos en este tipo de facies volcánicas, y como se depositan a lo largo de cauces y en cuencas alargadas, tienden lateralmente a desaparecer.

Las lavas del Miembro Los Bambinos han sido datadas de manera indirecta con radiocarbono en el sitio de la toma de la Fuente 29 (ver sitio en Fig. 2) de la Empresa de Servicios Públicos de Heredia (ESPH). Como se mencionó, un paquete de lahares subyacen a Los Bambinos Superior en este sitio y se encuentran entre las lavas de Los Bambinos Inferior y Superior hacia el este (Fig. 3). De ellos se ha obtenido un fragmento de madera semicarbonizada (posiblemente un aguacatillo, familia Lauracea, según I. Carpio, com. oral, 2006) de $135 \mathrm{~cm}$ x 30 $\mathrm{cm} \times 15 \mathrm{~cm}$ (Fig. 4), la cual ha sido datada con C-14 en Geochron Laboratories (muestra RCI170806-1GS, nominada en el laboratorio como GX-32662). La edad reportada es de $22500 \pm$ 310 años antes del presente (A.P.), la cual una vez calibrada con base en Van der Plicht et al.
(2004) arroja una edad de 27,4 \pm 0,4 ka (Fig. 4). Estrictamente, la edad es del lahar que contiene el tronco, mas puesto que el lahar se encuentra entre los sub-miembros de Los Bambinos, los cuales se supone no están separados por un largo periodo, se puede aproximar la edad de las lavas del Miembro Los Bambinos en 27,4 ka.

Las lavas del Miembro Bermúdez subyacen a los epiclastos del Miembro Carbonal en el río Ciruelas. Macroscópicamente son de color gris claro a rojizo, porfiríticas, con aprox. $15 \%$ de fenocristales de $3-4 \mathrm{~mm}$ (10\% de plagioclasas, $5 \%$ de piroxenos, $1 \%$ de olivino, con texturas glomeroporfiríticas, en donde las plagioclasas y los piroxenos contienen inclusiones de olivino), un $15 \%$ de microfenocristales de plagioclasa y olivino, y una matriz microcristalina de color gris claro. Son andesitas basálticas porfiríticas, diferentes de las lavas del Miembro Los Bambinos. Los mapas de BGS-SENARA (1985) y Protti (1986) no hicieron esta distinción petrográfica, y por eso en el río Ciruelas, esos autores continúan las lavas del Miembro Los Bambinos aguas abajo varios kilómetros (Fig. 1A). 


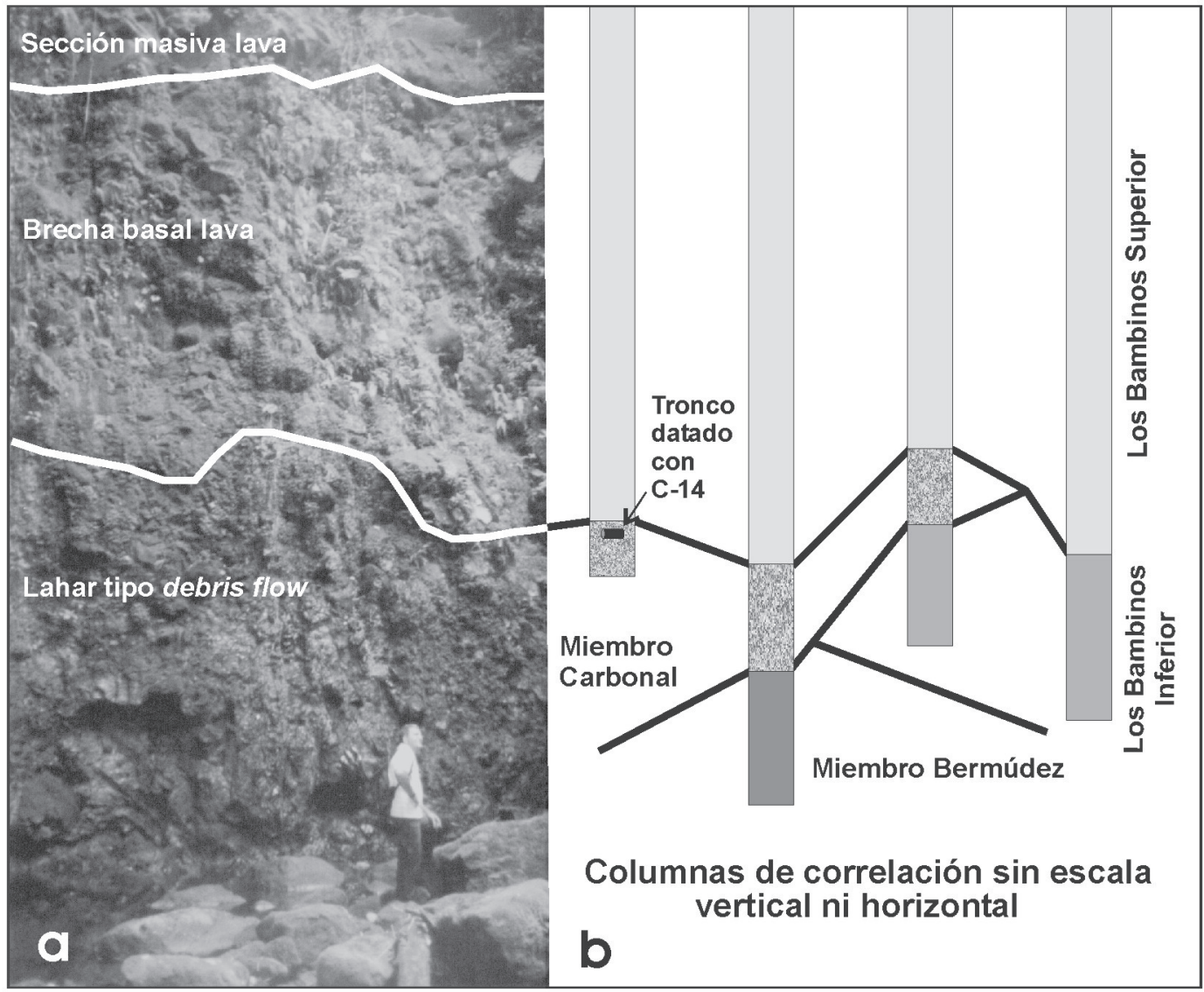

Fig. 3: a) Catarata en el río Ciruelas con la base de la lava de Los Bambinos Superior, contiguo a la toma 29 (231050N-524250E; ver sitio en Fig. 2). b) Correlación estratigráfica hacia el sector este con base en geología de campo y sondeos eléctricos verticales (GeoStratu Consultores S.A., 2005). Se muestra el sitio del muestreo para la datación del Miembro Los Bambinos (y Carbonal, detalle en Fig. 4).

\section{DISCUSIÓN: CORRELACIÓN ESTRATIGRÁFICA Y EDADES DE LA FORMACIÓN BARVA}

La geología de los pozos BA-175 y BA-403 (ubicación en Fig. 1) ha sido reinterpretada con base en los reportes existentes en el Archivo de Pozos de SENARA. La geología del pozo-piezómetro BA-809 ha sido reinterpretada a partir de la detallada descripción de los núcleos de perforación hecha por Arredondo (2003). Esta geología de pozos en la parte alta del volcán Barva (Fig. 1) junto con las observaciones geológicas apuntadas anteriormente, permiten una correlación geológica para esta parte del volcán y en general de la
Formación Barva (Figs. 5 y 6), y de lo cual se discute en los siguientes párrafos.

\section{El Proto Barva y el basamento del edificio del Barva}

Gans et al. (2003) han propuesto que una Proto Cordillera Central se desarrolló entre 0,98 y 0,85 Ma. No tenemos ningún argumento a favor o en contra de estas edades en este sector, porque no se han datado o mapeado. Sin embargo, Pérez et al. (2006) proponen que rocas sedimentarias se encuentran levantadas en el sector de La Hondura, al sureste de la caldera del Barva. También se han 


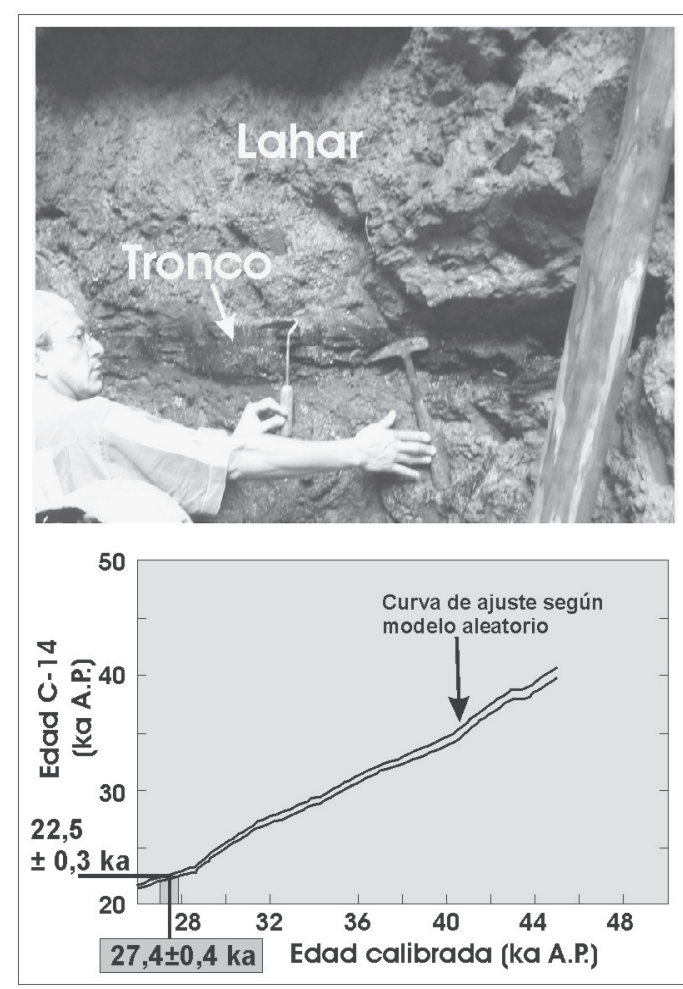

Fig. 4: Arriba, el tronco embebido dentro del lahar, que fue datado con C-14. Abajo, la calibración de la edad C-14 obtenida según el redibujo de la curva de Van der Plicht et al. (2004), que arroja una edad calibrada de 27,4 $\pm 0,4 \mathrm{ka}$.

encontrado fragmentos de rocas sedimentarias en ríos adyacentes a La Hondura y en el borde noreste del macizo volcánico, hacia Guápiles (T. Aguilar \& G. Peraldo, com. oral, 2004). Esto parece indicar que un basamento de sedimentitas neógenas y volcanitas pleistocenas existe bajo el Barva (Fig. 6).

Unas lavas cartografiadas en la Estación Biológica La Selva por Alvarado (1990), nominadas "Andesitas Esquina" y datadas en 1,2 $\pm 0,1$ Ma, bien podrían ser la base más antigua conocida del Proto Brava, pero faltan muchos más datos para soportar mejores ideas.

\section{El Paleo Barva (> 322 ka)}

Las rocas subyacentes a las ignimbritas de la Formación Tiribí en el piezómetro BA-809
(Figs. 5 y 6) son equivalentes estratigráficamente de forma lateral, por su posición infrayacente a Tiribí, con las rocas del Miembro Superior de la Formación Colima (edad 330 ka sensu Gans et al., 2003). No se tiene edad de ellas, pero puesto que subyacen a Tiribí (322 ka), se asume una edad > $322 \mathrm{ka}$. Por esto es que se les llama en este lugar como Paleo Barva.

Kussmaul (1988) propuso que las lavas de la Formación Colima "son el producto de efusiones a lo largo de fisuras con dirección NE-SW hasta E-W, o sea, aproximadamente paralelo al Río Virilla", en la falda de los estratovolcanes. Uno de los argumentos principales es una serie de diques visibles en el río Virilla, cerca de La Reforma, con esas direcciones. Sin embargo, tales diques son más bien de Colima Inferior o Miembro Belén, que subyacen asimismo a las ignimbritas de Puente de Mulas. Aún no se han publicado edades radiométricas de estas unidades, pero los datos de Gans et al. (2003) consignan tres ignimbritas del Valle Central con edades de 0,$57 ; 0,49$ y 0,44 Ma (esto es: 440-570 ka) y datos de Marshall et al. (2003) muestran que el Miembro Belén tendría $758 \pm 16$ ka. De tal manera, Colima Inferior es una secuencia de lavas y piroclastitas espesa depositadas en facies distales de la Paleocordillera Volcánica Central, que con probabilidad alcanza un espacio temporal amplio, sobreyacido por Puente de Mulas (y otras dos ignimbritas eruptadas desde la cordillera). Luego de Puente de Mulas, un periodo de pausa prolongado ( $\geq 110 \mathrm{ka}$ ), se dio en la parte distal, antes de que fueran depositadas las lavas de Colima Superior hace 330 ka. Se desconoce de dónde se originan las ignimbritas de Puente de Mulas, mas Colima Superior, con base en la distribución areal mostrada por Echandi (1981) en pozos y por Kussmaul (1988), parece más probable que las fracturas de las erupciones fisurales hayan sido paralelas al frente de la cordillera, más hacia el frente cordillerano que el presente río Virilla (Fig. 6), formando parte de la Paleo Cordillera Central, que había sido mencionada por Denyer et al. (1994) y Soto (1994).

Según Gans et al. (2003) esta Paleo Cordillera se formó entre 0,63-0,40 Ma, y la actual Cordillera se empezó a formar hace 0,17 Ma, pero habría que revisar ambos periodos. Carr et al. (2007) argumentan que el 


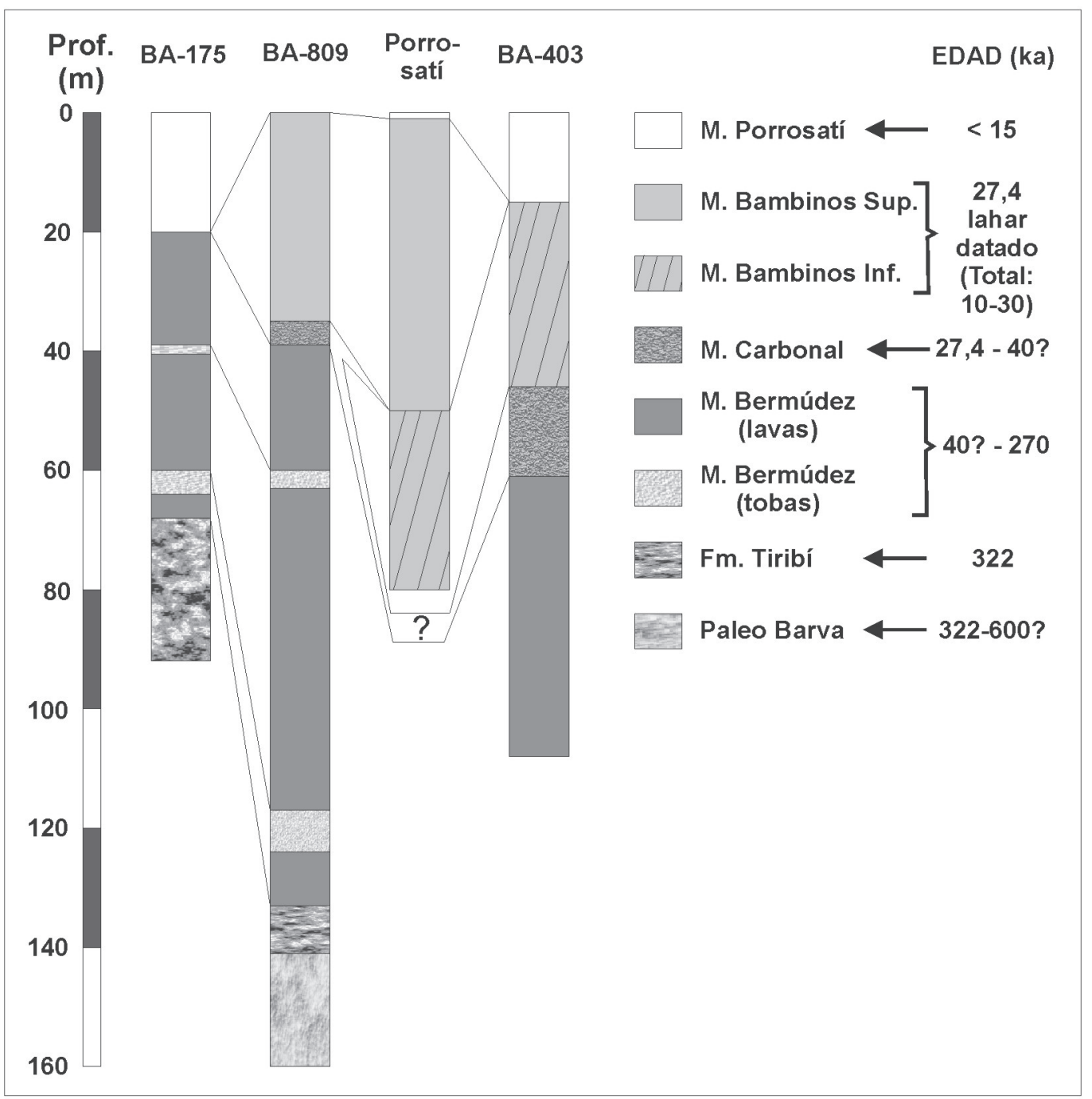

Fig. 5: Correlación estratigráfica entre los pozos BA-175, BA-403 y el piezómetro BA-809 (ubicación en la Fig. 1), con la geología de los alrededores de Porrosatí, y las edades discutidas en el texto, para los miembros de la Formación Barva.

presente arco volcánico se inició cerca de hace $600 \mathrm{ka}$ y continúa, pues han encontrado rocas con edades en todo ese lapso. Puesto que las evidencias mostradas por Pérez et al. (2006) y la geología del pozo-piezómetro BA-809 apuntan a que las ignimbritas de la Formación Tiribí se originaron en el Barva, y parece haber una diferencia morfológica y cronológica entre las ignimbritas y unidades sobreyacentes, se propone que el Paleo Barva incluya desde $600 \mathrm{ka}$ hasta el evento ignimbrítico, o sea hasta
322 ka. Los erosionados cerros Zurquí y periféricos, por ejemplo, serían los testigos laterales de la Paleo Cordillera (Alvarado, 1984; Soto, 1994; Denyer et al., 1994).

\section{La Formación Barva (el Neo Barva)}

La Formación Barva se ha visto como todas aquellas unidades sobreyacentes a la Formación Tiribí, con origen en el macizo del 


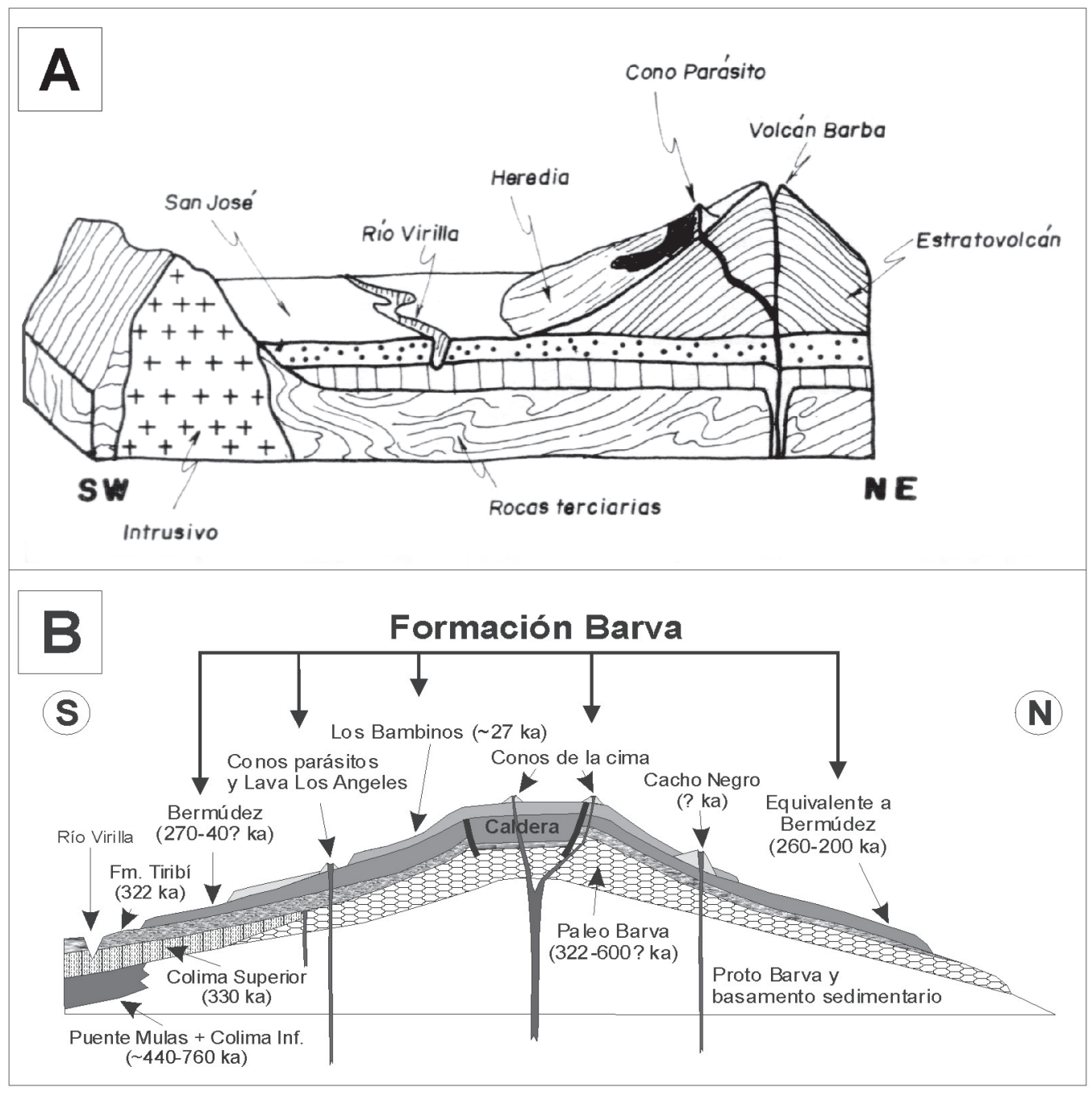

Fig. 6: Evolución del volcán Barva. A) Según lo propuesto por Protti (1986, Fig. 4), con un modelo simple y una edad del volcán de $30 \mathrm{ka}$. B) Un perfil reinterpretativo con base en la geología aquí discutida y las nuevas edades conocidas de las unidades, en donde el volcán tiene varias etapas, y la Formación Barva incluye la última (Neo Barva), desarrollada durante los últimos 270 ka. Ambos esquemas sin escala.

Barva (cf. Echandi, 1981; Protti, 1986), lo cual implica que esta formación es equivalente a la última etapa de evolución del volcán Barva, o sea Neo Barva. No se tienen muchas dataciones, pero la más vieja es de 270 ka (Pérez, 2000), por lo que se asume un cese del volcanismo entre 270-322 ka. Para efectos prácticos se conservan los nombres de las unidades propuestas por BGS-SENARA (1985) y Protti (1986) en esta discusión.

\section{Miembro Bermúdez}

Las rocas más distales del Barva, del Miembro Bermúdez cerca de Belén, que fueron datadas en 270 ka (Pérez, 2000) y que alcanzan hasta San Rafael de Alajuela y hasta Cebadilla, podrían ser también fisurales (serían el inicio del Neo Barva), debido a su extensión tan distal al volcán, aunque no hay evidencias de esto, porque están sepultadas por lavas más nuevas. 
En el sector septentrional del macizo se han datado cuatro lavas por Carr et al. (2007). Sus ubicaciones están en el sector norte de la hoja Poás y al sur de la hoja Río Cuarto, entre Ángeles, Colonia Carvajal y Magsasay. Cerca de allí, en la hoja Río Cuarto (coordenadas 258,7 N - 525,1 E, $400 \mathrm{~m}$ y 259,0 N - 523,65 E, $360 \mathrm{~m}$ ) afloran andesitas ácidas con alto potasio (Soto, 1999), casi totalmente afíricas, con pequeños fenocristales de plagioclasa en una matriz vítrea, que junto con otras rocas porfiríticas fueron asignadas por Soto (1999) como algunas de las rocas posiblemente más antiguas del Barva. Las nuevas edades mencionadas están en el rango entre $201 \pm 20$ ka y 262 $\pm 10 \mathrm{ka}$, de modo que serían equivalentes cronológicos con las distales del sector sur del macizo.

Las rocas de este Miembro hacia la Cordillera, sobreyacentes a las anteriores, en uno y otro sector, tendrían otros focos de emisión y podrían alcanzar edades de hasta unos $40 \mathrm{ka}$, antes de la efusión de las lavas de Los Bambinos, según algunas nuevas correlaciones estratigráficas presentadas por Vargas \& Acosta (2007). Como se ve en los pozos BA-175 y BA-809 (Fig. 5), hay en la parte superior al menos tres paquetes de lavas en el Miembro Bermúdez, separadas por tobas, y en las partes distales cerca de Belén otros paquetes similares, de modo que representan sin lugar a dudas, varios campos de coladas de lavas con un rango amplio de edades.

\section{Miembro Carbonal}

Los volcanes de la Cordillera Central son complejos con muchos cráteres en la cima y con conos piroclásticos sobre las faldas, compuestos principalmente por armazones de campos de coladas de lava. Los depósitos piroclásticos parecen estar subordinados en volumen, pues forman paquetes métricos que se adelgazan rápidamente, mientras las lavas son paquetes decamétricos con amplias extensiones, aunque representan eventos de corta duración. Las unidades piroclásticas, dependiendo de las coladas de lava subyacentes, pueden verse como eventos diacrónicos. Este es el caso del Miembro Carbonal, que como se mencionó supra, son piroclastos y epiclastos que en la parte superior del volcán puede tener edades en un rango de aprox. $40 \mathrm{ka}$ (cf. Vargas \& Acosta, 2007) y $27,4 \mathrm{ka}$, que es la edad de los lahares interdigitados entre las lavas de Los Bambinos superior e inferior.

\section{Miembro Los Bambinos}

El lahar entre las dos unidades superior e inferior se ha datado en 27,4 ka, de modo que las lavas inferiores son algo más viejas y las superiores algo más jóvenes, aunque se supone un lapso de algunos años a decenas de años de diferencia, entre sí. El campo total de coladas en toda su extensión mostrada en la figura 1B alcanza coladas en el sector noreste de la cima, hacia Sacramento, e incluso las coladas del cerro Guararí, como un modo de simplificación estratigráfica, aunque estas últimas han sido estimadas de haber sido eruptadas entre 8,2 y 13,4 ka por Vargas \& Acosta (2007). Así, sin tener más dataciones, el paquete de lavas de Los Bambinos se asigna con una edad aproximada de $27 \mathrm{ka}$, aunque puede tener un rango de 30-10 ka.

\section{Miembro Los Ángeles}

Las coladas del Miembro Los Ángeles (ver extremo inferior derecho de la Fig. 1B), puede tener una edad similar o inferior a Los Bambinos, a juzgar por su frescura morfológica y a que prácticamente no tienen ninguna cobertura de cenizas, aunque están en una posición fuera del eje máximo de caída de cenizas del Barva. Edades del orden de 10-15 ka parecen plausibles.

\section{Miembros Porrosatí y Cráter}

Por ser piroclastos relativamente proximales y recientes, se considra prudente unir ambos miembros en unos solo bajo el nombre Porrosatí. Brenes (2003) aportó una datación C-14 de tefras que sobreyacen a las lavas Los Bambinos en el camino entre Sacramento y la casa de guardaparques, con una edad de 11380 \pm 278 años, la cual hemos calibrado con base en Stuiver \& Reimer (2006) y se obtiene 13,4 
$\pm 0,4$ ka. Otra edad obtenida por G.E. Alvarado (com. oral, 2004) en unos piroclastos intermedios arrojan aprox. 8,2 ka, mientras que los piroclastos más recientes en la cima parecen tener cerca de 500 años. Por lo tanto, se considera entonces que los piroclastos del Miembro Porrosatí tendrían una edad aproximada de 15 ka o menos.

\section{CONCLUSIONES}

La reinterpretación de la geología de algunos pozos en la parte alta del volcán Barva, en conjunto con observaciones geofísicas, geológicas, dataciones previas y una nueva, y un mapeo de campo y fotogeológico-geomorfológico de parte de ese sector, permiten una correlación geológica de la Formación Barva y proponer una evolución global del macizo del Barva.

- Las rocas subyacentes a la Formación Tiribí en el piezómetro BA-809 son correlacionables estratigráficamente con la Formación Colima, formando parte de la Paleo Cordillera Central, de modo que el Paleo Barva incluiría a la Formación Tiribí.

- La Formación Barva es el equivalente al sector estratigráfico más nuevo del macizo del Barva, el Neo Barva, que se habría desarrollado en los últimos $270 \mathrm{ka}$.

- Las rocas más distales del Miembro Bermúdez cerca de Belén (270 ka), que alcanzan hasta San Rafael de Alajuela e incluso Cebadilla, podrían ser fisurales y serían el inicio del $\mathrm{Neo}$ Barva. En el sector norte, las más distales tienen edades similares (201-262 ka). Este miembro representa varios campos de coladas de lavas con un rango amplio de edades que alcanzaría hasta $\sim 40 \mathrm{ka}$.

- Los volcanes actuales de la Cordillera Central son complejos con depósitos piroclásticos subordinados en volumen que representan eventos de corta duración, que son eventos diacrónicos, como el caso del Miembro Carbonal, que en la parte superior del volcán puede tener edades en un rango de 40-27,4 ka.
- Las lavas del Miembro Los Bambinos se han dividido en dos sub-miembros (Inferior y Superior), y se han datado en 27,4 ka con base en un tronco de un lahar que divide ambos miembros, pero como forma un extenso campo de coladas en la parte superior del volcán, se estima que puede alcanzar edades de 10-30 ka en su conjunto.

- Los piroclastos del Miembro Porrosatí tendrían una edad menor que $15 \mathrm{ka}$, a juzgar por las edades de tefras mencionadas anteriormente, que sobreyacen a las lavas de Los Bambinos.

\section{AGRADECIMIENTOS}

Se agradece a Tecnoambiente Centroamericano S.A., por el financiamiento de la datación de C-14. A la ESPH por el apoyo en las investigaciones encaminadas a la prospección y explotación de aguas subterráneas, de lo cual este trabajo es un corolario. Este trabajo se enmarca como parte de las investigaciones del proyecto de tesis de Maestría en Geología con Énfasis en Aguas Subterráneas, de la primera autora, titulado "Perforación de pozos horizontales y evaluación del acuífero Bambinos Superior, para mejorar el abastecimiento y mitigar los racionamientos de agua potable en Heredia, Costa Rica", en proceso. Se agradecen los comentarios y sugerencias de Guillermo E. Alvarado y los revisores Esteban Gazel y Siegfried Kussmaul.

\section{REFERENCIAS}

ALVARADO, G.E., 1984: Aspectos petrológicos-geológicos de los volcanes y unidades lávicas del Cenozoico Superior de Costa Rica.- xii+183 págs. Univ. de Costa Rica, San José [Tesis Lic.].

ALVARADO, G.E., 1990: Características geológicas de la Estación Biológica La Selva, Costa Rica.- Tecnol. Marcha, 10 (3): 11-22.

ARREDONDO, S., 2003: Informe final del pozo de investigación.- 10 págs. Empresa perforadora Geoperforaciones, S.A.- 
Laboratorio de Hidrología Ambiental, UNA [Inf. Interno].

BGS \& SENARA, 1985: Mapa hidrogeológico del Valle Central de Costa Rica.- Escala 1:50 000, E.S.R. Limited, Inglaterra.

BOHNENBERGER, H., 1968: A photogeological study of the western Central Valley, Costa Rica.- 20 págs. Ministerio de Agricultura y Ganadería y Servicio Nacional de Acueductos y Alcantarillados, San José [Inf. Interno].

BOHNENBERGER, H. \& MADRIGAL, R., 1968: Mapa fotogeológico de la cuenca del río Virilla y sus alrededores.- Escala 1:50 000, Ministerio de Agricultura y Ganadería y Servicio Nacional de Acueductos y Alcantarillados, San José.

BRENES, J., 2003: Geología y peligro volcánicos del Flanco Oeste y Suroeste del volcán Barva.- 115 págs. Univ. de Costa Rica, San José [Tesis Lic.].

CARR, M.J., SAGINOR, I., ALVARADO, G.E., BOLGE, L.L., LINDSAY, F.N., MILIDAKIS, K., TURRIN, B.D., FEIGENSON, M.D. \& SWISHER III, C.C., 2007: Element fluxes from the volcanic front of Nicaragua and Costa Rica.Geochem. Geophys. Geosyst. 8(6), DOI: 10.1029/2006GC001396.

CROSBY, I.B., 1945: Geología del cañón del río Virilla, en la Meseta Central Occidental de Costa Rica.- Depto. Nacional de Agricultura, Boletín Técnico, 49: 217231.

DENGO, G. \& CHAVERRI, G., 1951: Reseña Geológica de la región Sudoeste de la Meseta Central de Costa Rica.- Rev. Univ. Costa Rica, 5: 313-326.

DENYER, P. \& ARIAS, O., 1990: Geología de la Hoja Abra.- Escala 1:50 000, Univ. de Costa Rica, Instituto Geográfico Nacional.
DENYER, P. \& ARIAS, O., 1991: Estratigrafía de la Región Central de Costa Rica.- Rev. Geol. Amér. Central, 12: 1-59.

DENYER, P., ARIAS, O. \& KUSSMAUL, S., 1994: Estratigrafía de las rocas ígneas.- En: DENYER, P. \& KUSSMAUL, S. (comps.): Atlas Geológico Gran Área Metropolitana. Edit. Tecnológica de Costa Rica: 61-70.

ECHANDI, E., 1981: Unidades volcánicas de la vertiente norte de la cuenca del río Virilla.123 págs. Univ. de Costa Rica, San José [Tesis Lic.].

FERNÁNDEZ, M., 1969: Las unidades hidrogeológicas y los manantiales de la vertiente norte de la cuenca del río Virilla. - Investigaciones de aguas subterráneas en Costa Rica. Informe Técnico, 27: 1-56.

GANS, P.B., ALVARADO, G., PÉREZ, W., MACMILLAN, I., \& CALVERT, A., 2003: Neogene Evolution of the Costa Rican Arc and Development of the Cordillera Central.- Abstract, Geological Society of America, Cordilleran Section, 99th Annual, April 2003.

GEOSTRATU CONSULTORES S.A., 2005: Estudio geofísico mediante resistividad eléctrica para ESPH en Los Bambinos de San José de la Montaña, Barba, Provincia de Heredia.- 18 págs. [Inf. Interno].

KUSSMAUL, S., 1988: Comparación petrológica entre el piso volcánico del Valle Central y la Cordillera Central de Costa Rica.Ciencia y Tecnología 12(1-2): 109-116.

MARSHALL,J.S.,IDLEMAN,B.D.,GARDNER, T.W. \& FISHER, D.M., 2003: Landscape evolution within a retreating volcanic arc, Costa Rica, Central America.- Geology, 31, 5: 419-422.

PÉREZ, W., 2000: Vulcanología y petroquímica del evento ignimbrítico del Pleistoceno Medio (0,33 M.a.) del Valle Central de 
Costa Rica.- 170 págs. + apéndices. Univ. de Costa Rica, San José [Tesis Lic.].

PÉREZ, W., ALVARADO, G.E. \& GANS, P., 2006: The 322 ka Tiribí Tuff: stratigraphy, geochronology and mechanisms of deposition of the largest and most recent ignimbrite in the Central Valley, Costa Rica.- Bul. Volcanol. 69: 25-40.

PROTTI, R., 1986: Geología del flanco sur del volcán Barva.- Bol. Vulcanol. UNA, 17:23-31.

SCHAUFELBERGER, P., 1932: Un estudio elemental sobre la geología de Costa Rica.- La Escuela Costarricense 1-3: 156 , San José.

SOTO, G.J., 1994: Volcanología Física.En: DENYER, P. \& KUSSMAUL, S. (comps.): Atlas Geológico Gran Área Metropolitana. Edit. Tecnológica de Costa Rica: 131-146.

SOTO, G.J., 1999: Geología Regional de la Hoja Poás (1:50 000).- En: ALVARADO, G.E. \& MADRIGAL, L.A. (Eds.): Estudio GeológicoGeotécnico de Avance a la Factibilidad del P.H. Laguna Hule.- ICE, San José, pp. 15-45 [Inf. interno].

STUIVER, M. \& REIMER, P., 2006: CALIB 5.1.0beta.- http://calib.qub.ac.uk/calib/

VAN DER PLICHT, J., BECK, J.W., BARD, E., BAILLIE, M.G.L., BLACKWELL, P.G., BUCK, C.E., FRIEDRICH, M., GUILDERSON, T.P., HUGHEN, K.A., KROMER, B., MCCORMAC, F.G., BRONK RAMSEY, C., REIMER, P.J., REIMER, R.W., REMMELE, S., RICHARDS, D.A., SOUTHON, J.R., STUIVER, M. \& WEYHENMEYER, C.E., 2004: NotCal04-Comparison/ Calibration 14C Records 26-50 cal kyr BP.- Radiocarbon, 46(3): 1225-1238.

VARGAS, C.A. \& ACOSTA, P., 2007: Tephrastratigraphy and volcanic hazards for the upper part of Barva volcano, Costa Rica.- Abstract, Workshop to Integrate Subduction Factory and Seismogenic Zone Studies in Central America, Heredia, Costa Rica, junio del 2007, p. 1.

WILLIAMS, H., 1952: Volcanic history of the Meseta Central Occidental, Costa Rica.Univ. Calif. Pub. Geol. Sc. 29(4): 145-180. 
